\title{
Practical Implementation of Diabetes Technology: It Is Time
}

\author{
Laurel H. Messer
}

Introduction

$\mathbf{T}$ HIS YEAR, THE ATTD YEARBOOK debuts a new category: Practical Implementation of Diabetes Technology. In the span of one decade, dozens of diabetes technologies have been approved for diabetes care that were previously unavailable. Devices are sleeker, more accurate and intelligent, better looking, and simply do more than ever before. Access has increased to unprecedented levels worldwide, though there is more work to be done.

Practical implementation of technology encompasses several related domains of inquiry, including clinical utility and guidance, education, economics and access to technology, benefits/barriers, and "real-world" use (Fig. 1). It extends across the five types of technology, three pharmacological treatments, and evaluation of human factors covered in this yearbook, but with a singular focus: real-world use. We frequently study how technology could or should be utilized but often do not understand how technology is being utilized. The requisite questions of "real-world" technology use include: Who will use it? How will they use it? Why do they use it? How can we help them use it? This article represents the "So what?" of technological advancements for treatment of diabetes.

The 2018 review for this article includes research articles retrieved from PubMed dating from July 2017 to June 2018, using search terms around diabetes technology, including "education," "access," "economics," "real world," "behavior," "attitudes," "expectations," and "practical implementation." Of the 41 retrieved articles, 9 are highlighted here.

There are three prominent themes highlighted in this year's literature review:

1. Use of technology in the real world: How diabetes technology is being used by individuals outside of structured research studies. This includes novel uses of remote monitoring in a school setting, do-it-yourself technologies, and clinical outcomes of expanding access for continuous glucose monitoring (CGM) wear.

2. Practical challenges and solutions with diabetes technology: Current research characterizing challenges and offering solutions to the burdens of using diabetes technology. This year includes focus on skin issues, infusion set failure, and targeted education for insulin pump use.

3. Access and cost-effectiveness of technology: The consequences of access to diabetes technology across the world, as well as cost-effectiveness evaluation for real-world diabetes care. This includes a report of insulin management practices for new onset type 1 diabetes (T1D), and cost effectiveness of CGM for different populations.

As in silico and controlled clinical trial milestones are reached, the new work begins: understanding what happens when we release diabetes technology into the wild. We, the scientific community, can offer the ultimate service to the diabetes community by equipping individuals with the right therapies, the right education, and the right support. It is crucial we understand how to do so. Practical implementation of diabetes technology is a discipline devoted to these causes. It is imperative that the academic literature expands to inform this mission.

Barbara Davis Center for Diabetes and University of Colorado School of Medicine, Aurora, CO 
Key Articles Reviewed for the Article

Effect of continuous glucose monitoring on glycemic control, acute admissions, and quality of life: a real-world study

Charleer S, Mathieu C, Nobels F, De Block C, Radermecker RP, Hermans MP, Taes Y, Vercammen C, T'Sjoen G, Crenier L, Fieuws S, Keymeulen B, Gillard P; for RESCUE

Trial Investigators

J Clin Endocrinol Metab 2018; 103: 1224-1232

Real-world use and self-reported health outcomes of a patient-designed do-it-yourself mobile technology system for diabetes: lessons for mobile health

Lee JM, Newman MW, Gebremariam A, Choi P, Lewis D, Nordgren W, Costik J, Wedding $J$, West B, Gilby NB, Hannemann C, Pasek J, Garrity A, Hirschfeld E

Diabetes Technol Ther 2017; 19: 209-219

Schooling diabetes: use of continuous glucose monitoring and remote monitors in the home and school settings

Erie C, Van Name MA, Weyman K, Weinzimer SA, Finnegan J, Sikes K, Tamborlane WV, Sherr $J L$

Pediatr Diabetes 2018; 19: 92-97

High frequencies of dermatological complications in children using insulin pumps or sensors

Berg AK, Olsen BS, Thyssen JP, Zachariae C, Simonsen AB, Pilgaard K, Svensson $J$

Pediatr Diabetes 2018; 19: 733-740

Insulin pump breakdown and infusion set failure in Italian children with type 1 diabetes: 1-year prospective observational study with suggestions to minimize clinical impact

Rabbone I, Minuto N, Toni S, Lombardo F, Iafusco D, Marigliano M, Schiaffini R, Maltoni $G$, Frongia AP, Scardapane M, Nicolucci A, Cherubini V, Bonfanti R, Scaramuzza AE; for the Diabetes Study Group of the Italian Society of Pediatric Endocrinology and Diabetology

Diabetes Obes Metab 2018; 20: 2551-2556

Impact of targeted education on managing warning and error signals by children and adolescents with type 1 diabetes using the Accu-Chek Combo Insulin Pump System

Deeb A, Qahtani NA, Ali AA, Akle M

Ann Pediatr Endocrinol Metab 2017; 22: 170-175

Insulin regimens for newly diagnosed children with type 1 diabetes mellitus in Australia and New Zealand: a survey of current practice

Selvakumar D, Al-Sallami HS, de Bock M, Ambler GR, Benitez-Aguirre P, Wiltshire E, Tham E, Simm P, Conwell LS, Carter PJ, Albert BB, Willis J, Wheeler BJ; on behalf of the Paediatric Society of New Zealand Diabetes Clinical Network

J Paediatr Child Health 2017; 53: 1208-1214

Cost-effectiveness of continuous glucose monitoring for adults with type 1 diabetes compared with self-monitoring of blood glucose: the DIAMOND randomized trial

Wan W, Skandari MR, Minc A, Nathan AG, Winn A, Zarei P, O’Grady M, Huang ES

Diabetes Care 2018; 41: 1227-1234

Cost-effectiveness of insulin pumps compared with multiple daily injections both provided with structured education for adults with type 1 diabetes: a health economic analysis of the Relative Effectiveness of Pumps over Structured Education (REPOSE) randomised controlled trial

Pollard DJ, Brennan A, Dixon S, Waugh N, Elliott J, Heller S, Lee E, Campbell M, Basarir $H$, White $D$; on behalf of the REPOSE group 


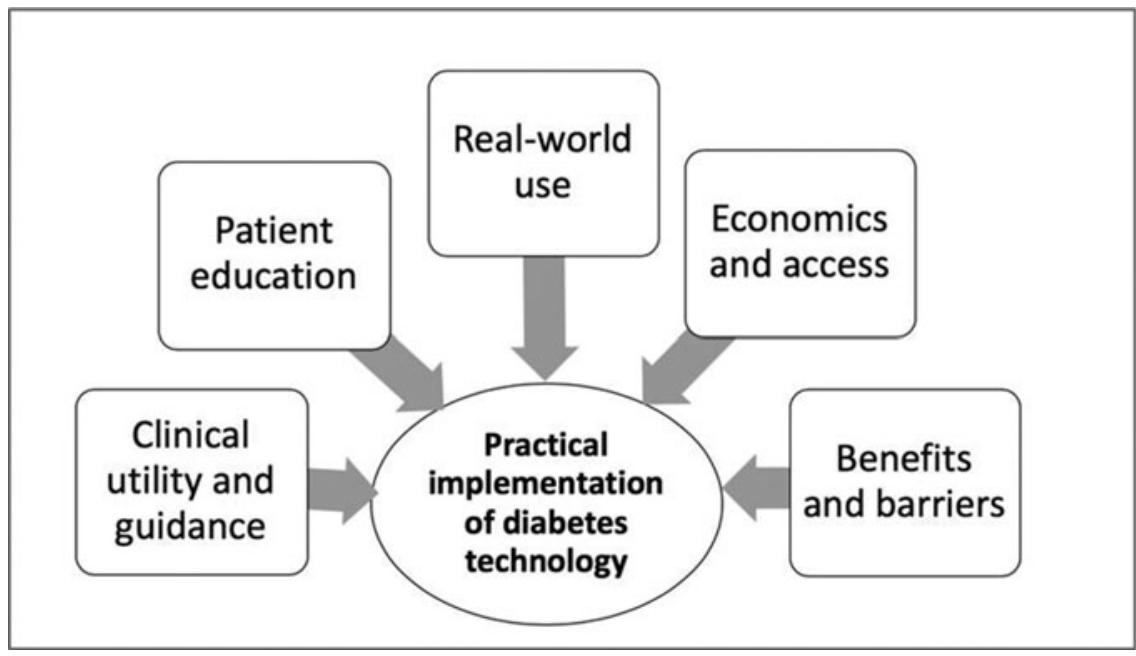

FIG. 1. Domains of inquiry-related practical implementation of diabetes technology.

\section{REAL-WORLD TECHNOLOGY USE}

\section{Effect of continuous glucose monitoring on glycemic control, acute admissions, and quality of life: a real-world study}

Charleer $S^{1,2}$, Mathieu $C^{1}$, Nobels $F^{3}$, De Block $C^{4}$, Radermecker $R P^{5}$, Hermans $M P^{6}$, Taes $Y^{7}$, Vercammen $C^{8}$, T'Sjoen $G^{9}$, Crenier $L^{10}$, Fieuws $S^{11}$, Keymeulen $B^{12}$, Gillard $P^{1}$; for RESCUE Trial Investigators

${ }^{1}$ Department of Endocrinology, University Hospitals Leuven-Katholieke Universiteit Leuven, Leuven, Belgium; ${ }^{2}$ PhD Fellowship Strategic Basic Research of the Research Foundation-Flanders (Fonds Wetenschappelijk Onderzoek), Brussels, Belgium; ${ }^{3}$ Department of Endocrinology, Onze-Lieve-Vrouw Hospital Aalst, Aalst, Belgium; ${ }^{4}$ Department of Endocrinology, Diabetology and Metabolism, University of Antwerp-Antwerp University Hospital, Antwerp, Belgium; ${ }^{5}$ Department of Diabetes, Nutrition and Metabolic Disorders, Centre Hospitalier Universitaire Liege-Liege University, Liege, Belgium; ${ }^{6}$ Department of Endocrinology and Nutrition, Cliniques Universitaires St-Luc-Université Catholique de Louvain, Brussels, Belgium; ${ }^{7}$ Department of Endocrinology, Algemeen Ziekenhuis Bruges, Belgium; ${ }^{8}$ Department of Endocrinology, Imelda Hospital Bonheiden, Bonheiden, Belgium; ${ }^{9}$ Department of Endocrinology, Ghent University Hospital, Ghent, Belgium; ${ }^{10}$ Department of Endocrinology, Université Libre de Bruxelles-Hopital Erasme, Brussels, Belgium; ${ }^{11}$ Department of Public Health and Primary Care, I-BioStat, $K U$ Leuven-University of Leuven and Universiteit Hasselt, Leuven, Belgium; ${ }^{12}$ Diabeteskliniek, University Hospital Brussels-Vrije Universiteit Brussel, Brussels, Belgium

\section{J Clin Endocrinol Metab 2018; 103: 1224-1232}

This manuscript is also discussed in the article on Continuous Glucose Monitoring in 2018, page S-13, and the article on Technology and Pregnancy, page S-101.

\section{Background}

Studies on real-world use of CGM are few. CGM use is varied by country and region, often due to reimbursement and cost issues. The Belgium health-care authority instituted a 3year pilot program to provide reimbursement for CGM for individuals with T1D in an effort to analyze real-world outcomes of CGM: the Reimbursement Study of Continuous Glucose Monitoring in Belgium (RESCUE) trial.

\section{Methods}

This is an observational prospective cohort study of patients with T1D across 17 centers in Belgium. Patients were selected at the discretion of the diabetes providers if they had T1D for $>1$ year; used insulin pump > 6 months; demonstrated suboptimal glycemic control, uncontrolled hypoglycemia, or pregnancy; and expressed interest in wearing CGM. CGM was offered with complete financial reimbursement. Primary outcome was change in glycated hemoglobin (HbA1c) since initiation of CGM and secondary outcomes included admissions for acute diabetes complications, work absenteeism, quality of life (QOL), glycemic variability, and hypoglycemia.

\section{Results}

During the study period, 515 adults with T1D (59\% female) started CGM, and $81 \%$ continued for at least 12 months. HbA1c decreased from $7.7 \pm 0.9 \%(61 \pm 9.8 \mathrm{mmol} /$ $\mathrm{mol})$ to $7.4 \pm 0.8 \%(57 \pm 8.7 \mathrm{mmol} / \mathrm{mol})$ at 12 months $(P<0.001)$. Pre-post analysis of the year before and after CGM initiation showed reduction in hospitalizations from $16 \%$ of individuals to $4 \%$, and decreased admission days from 54 to 18 per 100 patient-years $(P<0.001)$. These reductions were seen for both hypoglycemia and diabetic ketoacidosis admissions. Work absenteeism likewise decreased from one quarter of individuals reporting missed work in the year prior to CGM to $9 \%$ of individuals missing work after CGM initiation. QOL measures showed improvement from baseline to 12 months on diabetes-specific distress, fear of hypoglycemia, general health, and social functioning. Those who initiated CGM due to hypoglycemia experienced the largest QOL improvement. Hypoglycemia decreased from a mean of $11.1 \pm 5.7 \%$ via self-monitoring of blood glucose (SMBG) prior to CGM to $4.5 \pm 3.2 \%$ after 12 months $(P=0.002)$. 


\section{Conclusion}

Over a 12-month period, HbAlc decreased with the addition of CGM in adult patients with T1D using insulin pumps. Quality of life was improved while diabetes-related hospitalizations and workplace absenteeism decreased. CGM showed glycemic and QOL benefits in real-world clinical care.

\section{Comments}

Real-world outcomes should affect how we deliver, pay for, and administer diabetes care. This article by Charleer and colleagues illustrates how systematic access to diabetes technology can affect outcomes for individuals with diabetes. The country of Belgium piloted a program to provide CGM free of cost to adults with T1D with suboptimal glycemic control at 17 different clinical centers. Not surprisingly, both glycemic and qualityof-life outcomes were improved in the clinical CGM population. Clinicians should consider the following implications:

- Although this program relied heavily on physician discretion for CGM candidate selection, the study design and population represent real-world effectiveness trial more than an efficacy trial.

- Reduced hospital admissions, shorter hospital admissions, and work absenteeism decreased significantly after starting CGM. These outcomes carry more weight in this particular trial because they are extracted from a 12-month period, which is relatively long-term for CGM trials. Tangible benefits like these should be discussed with patients considering CGM. These metrics are also more useful to payers than other self-report QOL metrics.

- We applaud the Belgium system for experimenting with reimbursement for CGM and hope other countries and systems will consider similar trials. Will the health coverage for CGM in Belgium change because of this trial? Will it be expanded to other populations (i.e., children)? These are important systematic follow-up questions to be answered.
Real-world use and self-reported health outcomes of a patient-designed do-it-yourself mobile technology system for diabetes: lessons for mobile health

Lee $J M^{1,2}$, Newman $M W^{3}$, Gebremariam $A^{2}$, Choi $P^{2}$, Lewis $D^{4}$, Nordgren $W^{5}$, Costik $J^{6}$, Wedding $J^{5}$, West $B^{7}$, Gilby $\mathrm{NB}^{3}$, Hannemann $\mathrm{C}^{7}$, Pasek $\mathrm{J}^{8}$, Garrity $A^{2}$, Hirschfeld $E^{2}$

${ }^{I}$ Pediatric Endocrinology, University of Michigan, Ann Arbor, MI; ${ }^{2}$ Child Health Evaluation and Research Center, University of Michigan, Ann Arbor, MI; ${ }^{3}$ School of Information, University of Michigan, Ann Arbor, MI; ${ }^{4}$ \#OpenAPS, Seattle, WA; ${ }^{5}$ The Nightscout Foundation, McKinney, TX; ${ }^{6}$ Center for Clinical Innovation, University of Rochester, Rochester, NY; ${ }^{7}$ Nightscout Community, San Francisco, CA; ${ }^{8}$ Communication Studies and Center for Political Studies, Institute for Social Research, University of Michigan, Ann Arbor, MI

Diabetes Technol Ther 2017; 19: 209-219

\section{Background}

Do-it-yourself (DIY) mobile technologies for diabetes are being used by people with diabetes in order to remotely monitor glucose levels or employ closed-loop insulin delivery in non-commercially approved platforms. Little is known about DIY users as the community primarily exists on social media (CGM in the Cloud Facebook group), and the practice is not formally recommended or tracked by regulatory or clinical agencies.

\section{Methods}

This is a cross-sectional, household level online survey of members of the CGM in the Cloud Facebook group. Respondents answered questions about demographics, diabetes management, use of the Nightscout DIY remote monitoring technologies, perceived quality of life, and glycemic benefits.

\section{Results}

Of the 1268 members of CGM in the Cloud surveyed, 1157 individuals with diabetes were represented, $75 \%$ of which
FIG. 2. Nightscout use by age and reason according to sampled individuals (percentage).

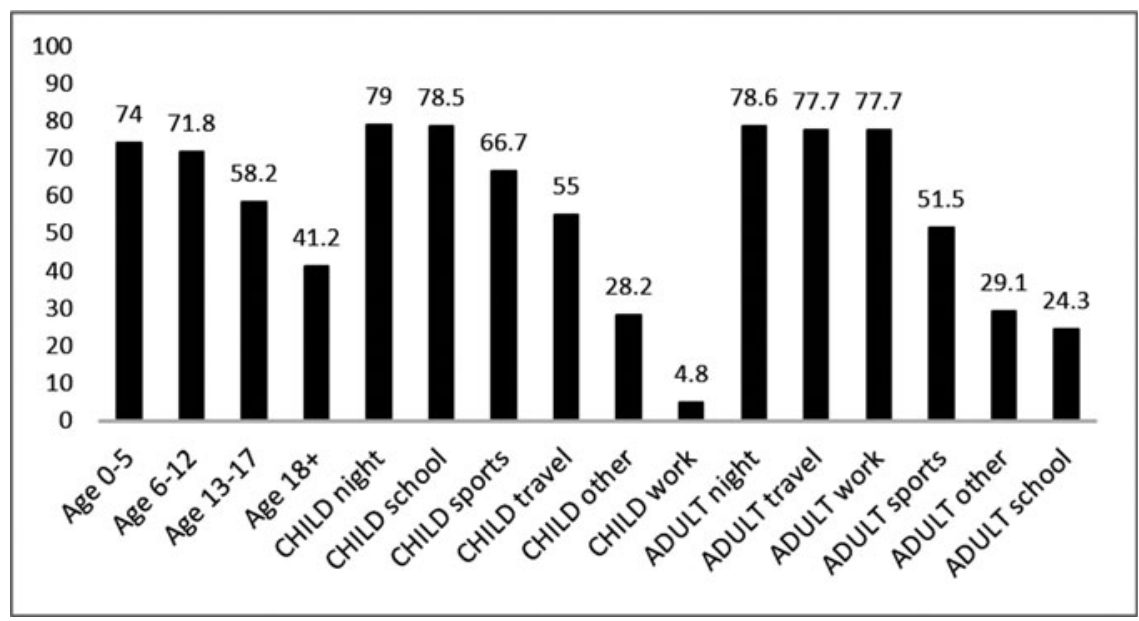


were children $<18$ years old. The majority were nonHispanic white $(90.2 \%)$ with type 1 diabetes $(99.4 \%)$ living in the United States (78\%) with private health insurance (83.8\%), using insulin pump (86\%) and CGM (97\%). Over half of the respondents reported using Nightscout (62\%). When broken down by age, the highest proportion of use was for children ages 6-12 (74\%), and 0-5 years (74\%), with $41.2 \%$ of adults using Nightscout for themselves (Fig. 2). The majority were for children ages 6-12 (51.2\%), and ages 0-5 years $(18.7 \%)$, with the lowest use in adolescents and adults (15\% each). A significant portion (18.5\%) of users reported adapting CGM technology specifically for Nightscout use. Caregivers/parents cited the most common reasons for use to be nighttime and school, and adults with diabetes similarly cited nighttime, work, and travel as most common reasons (Fig. 2). Pediatric users had a mean of three individuals viewing their data, while adults primarily viewed their own data. Nightscout users $(n=724)$ reported checking blood glucose levels less, bolusing more often, and improving HbA1c when compared to period prior to Nightscout use.

\section{Conclusion}

DIY Nightscout is currently being used by pediatric and adult patients with diabetes and shows improved outcomes by self-report of users.

\section{Comments}

Data is starting to emerge from the do-it-yourself (DIY) community. This article from Lee et al. is the first to present data on the DIY Nightscout technology, which allows for remote monitoring of CGMs not equipped with factory-provided remote monitoring. Regardless of the outlook and sentiment of the scientific community on DIY, it is incumbent to understand how persons with diabetes are using DIY systems in the real world. This article elucidates the following:

- Parents of children with T1D and adults with T1D both use Nightscout remote monitoring for similar reasons: parents for the overnight period, school, and sporting events, while adults with T1D for overnight period, work, and travel. These are periods with reduced awareness or less attention to diabetes, and when additional tools for monitoring enhance sense of safety.

- It is interesting to note that $18 \%$ of the sample were motivated to initiate CGM in order to use the Nightscout. Put another way, CGM may not have been considered by some of these individuals/families except for the ability to remotely monitor glucose levels. This is a crucial message for industry to hear: Patients with T1D desire remote monitoring for their devices, especially children and families. Every future glucose monitoring device for diabetes should include remote monitoring as a standard feature.

- In this sample, $75 \%$ of the T1D individuals were children/adolescents, an age when parents desire closer supervision of glucose levels. This likely represents the larger Nightscout population as well and not just this study. It would be interesting to note the demographics difference between DIY remote monitoring and DIY closed-loop technologies.

- There is tentative evidence in this article of increased quality of life, less blood glucose checking, and increased insulin dosing with Nightscout, though more rigorous longitudinal studies with validated metrics are needed to confirm this. Likewise, selfreported HbAlc was improved with Nightscout, but this was not collected in a rigorous manner. It is challenging to study the DIY phenomenon, as the nature of DIY is fundamentally grassroots, unregulated, and individual. Partners like JDRF may help accelerate research on these technologies (1).

\section{Schooling diabetes: use of continuous glucose monitoring and remote monitors in the home and school settings}

Erie $C^{1}$, Van Name $M A^{2}$, Weyman $K^{2}$, Weinzimer $S A^{2}$, Finnegan $J^{2}$, Sikes $K^{2}$, Tamborlane $W V^{2}$, Sherr $J^{2}$

${ }^{1}$ Charles E. Schmidt College of Medicine, Florida Atlantic University, Boca Raton, FL; ${ }^{2}$ Department of Pediatrics, Yale School of Medicine, New Haven, CT

Pediatr Diabetes 2018; 19: 92-97

This manuscript is discussed also in the article on Diabetes Technology and the Human Factor, page S-138.

\section{Background}

Continuous glucose monitors (CGM) and CGM with remote monitoring capabilities (CGM/RM) are often used for management of children with T1D both in the school and home setting. Although these devices are more commonly incorporated into the school setting, common practices by family and youth have not been well described. This study examined real-world CGM/RM use in school and home setting, as well as expectations and attitudes of parents and youth toward the technology.

\section{Methods}

Parents of pediatric patients at an academic diabetes center in the United States were surveyed on their child's use of CGM/RM, alert settings, frequency of data review, expectation of daytime caregivers (i.e., school personnel), and open-ended questions relating to attitudes and expectations of CGM/RM. Additionally, a separate survey was given to daytime caregivers to complete and mail back anonymously to the study center. The study used mixed methods analysis and grounded theory methodology for qualitative analysis.

\section{Results}

Thirty-three parent surveys and 17 daytime caregiver surveys were returned, with mean age of child being cared for of $9.1 \pm 4.0$ years old (range $2-17$ ). Thirty-two youth wore Dexcom G4 or G5, and one wore a Medtronic Enlite sensor. Parents reported $94 \%$ of youth wore the sensor 7 days/week, and $68 \%$ of them used remote monitoring capabilities, which was more common in the elementary school children $(81 \%)$ compared with middle and high school youth (50\%). All 
youth $(100 \%)$ had low glucose alerts activated on their CGM, and $97 \%$ used high glucose alert as well. Rate of fall/rise were used by $55 \% / 48 \%$ respectively. Both caregivers $(35 \%)$ and parents $(39 \%)$ indicated they had treated hypoglycemia without checking a blood glucose level based on the sensor alerts alone. Parents reported checking the remote monitoring system constantly (30\%) and hourly (26\%) more than any other time interval. The majority of parent respondents $(62 \%)$ indicated they downloaded the retrospective data quarterly or less, with $19 \%$ doing it monthly. Themes from qualitative analysis related to CGM and remote monitoring included peace of mind/security, increased child independence, and overall satisfaction with CGM.

\section{Conclusion}

CGM and remote monitoring is an important tool utilized in the home and school setting for youth with T1D and assists in actively managing diabetes care (especially hypoglycemia) by parents and caregivers alike.

\section{Comments}

This article by Erie et al. addresses the use of CGM/ remote monitoring (CGM/RM) with children at school. It has not been well characterized in the literature how school personnel and parents use CGM/RM during daytime hours. This study reveals some important clinical pearls for clinicians to consider:

- CGM/RM can be helpful for identifying and treating hypoglycemia in school and at home. Now that the U.S. FDA has approved nonadjunctive use of CGM for diabetes management, many more youth and caregivers will treat hypoglycemia based on sensor readings, reducing burden for all parties.

- Rate of change alerts are utilized substantially less than threshold alerts, and turning them off may be a good strategy to prevent alarm fatigue. High glucose alerts are likewise utilized less than low alerts, as acute hyperglycemia is not as immediately critical. Alerts on CGM and remote monitoring should be actionable alerts, and minimizing nuisance alerts is important.

- Remote monitoring allowed increased collaboration of parents and caregivers, who both reported feeling more secure and having "peace of mind" from being able to see the CGM data remotely. This may increase access to school environments for some families or improve communication between school personnel and parents for those who already attend school.

- CGM appears to be infrequently downloaded at home. This is not surprising and corroborates past data $(2,3)$. Although retrospective analysis is associated with better glycemic control (2), we do not know whether it actively contributes to better glycemic control, and it should be studied in a prospective manner.

- With the future increase of perpetual connectivity of diabetes devices, it will be interesting to see how school and community resources cope. Will future connected devices make diabetes management at school easier or more complicated from a school, home, and child perspective?

\section{PRACTICAL CHALLENGES AND SOLUTIONS}

\section{High frequencies of dermatological complications in children using insulin pumps or sensors}

Berg $A K^{1,2}$, Olsen $B S^{1}$, Thyssen $J^{3}$, Zachariae $C^{3}$, Simonsen $A B^{3}$, Pilgaard $K^{4}$, Svensson $J^{1,2}$

${ }^{1}$ Department of Pediatrics, Copenhagen Diabetes Research Center (CPH-DIRECT), Copenhagen University Hospital, Copenhagen, Denmark; ${ }^{2}$ Faculty of Health and Medical Science, University of Copenhagen, Copenhagen, Denmark; ${ }^{3}$ Department of Dermatology and Allergy, Copenhagen University Hospital, Copenhagen, Denmark; ${ }^{4}$ Pediatric and Adolescent Department, Nordsjallands Hospital, Hillerød, Denmark

Pediatr Diabetes 2018; 19: 733-740

\section{Background}

Dermatological complications related to insulin pump (continuous subcutaneous insulin infusion [CSII]) and CGM use have not been well characterized in children and adolescents with diabetes.

\section{Methods}

This was a cross-sectional study conducted in 144 patients/ parents from two outpatient diabetes clinics in Denmark. Parents of patients aged 2 to 11 years and patients aged 12 to 20 years old who were treated with insulin pump or CGM for more than 1 month were surveyed about diabetes characteristics, dermatological and allergy history, and details about current or previous dermatological complications from use of diabetes technology. Photographs and descriptions of types of dermatological complications were used to help solicit accurate descriptions from subjects.

\section{Results}

Mean pump use was 3.94 years. Of the 143 patients using insulin pump, $89.5 \%(\mathrm{CI}=85-95)$ had experienced complications related to pump use including itching (77.6\%), wet or dry wounds (50.3\%), and eczema (46.2\%); and 62.9\% had a current visible dermatological condition including eczema $(25.9 \%), \mathrm{red} / \mathrm{blue}$ dots at site of former insertion $(23.1 \%)$, and dry wounds (13.3\%) (Fig. 3). Lipohypertrophy and lipoatrophy were rare $(<3 \%)$. Using univariate logistic regression, the odds of dermatological complications were 3.7 times higher for those with a history of atopic disease ([95\% CI 1.49.7] $P<0.01)$ and 7.3 times higher for those using barrier cream ([CI 1.6-35.6], $P<0.01$ ). For CGM use, 66 of the 83 users had experienced complications including itching $(70 \%)$, eczema (46\%), and wounds (33\%). Seventy-six participants were currently using CGM and reported a variety of complications, shown in Fig. 3. For both insulin pump and CGM use, the odds of skin reactions were significantly less for those who used the devices less than 1 year $(0.44$ and 0.3 , respectively, $P<0.05)$. The mean score for discomfort from skin reactions was significantly lower for insulin pump reactions compared to CGM reactions $(P<0.01)$, with $38.5 \%$ of CGM users and $18.1 \%$ of pump users rating skin reactions as a "big problem" or "very big problem." 


\section{Conclusion}

Dermatological complications from device wear are highly prevalent and bothersome to pediatric patients with diabetes. This cross-sectional data highlights the need for a prospective study of dermatological conditions over time to elucidate prevention and treatment paths for clinicians to employ.

\section{Comments}

On-skin diabetes technologies present a host of issues related to skin integrity and site selection. Although this is well known in clinical practice, this Danish study in individuals using insulin pumps and CGM provides quantified information on a moderate-sized pediatric sample. Clinicians and researchers need to consider the following:

- There is high prevalence of skin issues with both insulin pumps and CGM use, but very little research into how to best address these issues. Over $70 \%$ of this sample reported experiencing skin reactions from either pump or sensor. Adults experience a high prevalence of reactions as well (4).

- Although atopic history and barrier cream were associated with increased prevalence of skin reactions (odds ratio 3.7 and 7.3 , respectively, $P<0.01$ ), this cross-sectional study cannot attribute causation. The authors point out the likely case that those who use barrier cream are doing so because of already present skin reactivity. Atopy has been previously associated with device reactions (5).

- There are no clinical practice guidelines recommending skin assessment for chronic device use: there are guidelines for skin inspection related to statin and niacin use (6), foot care (7), and peripheral neuropathy (8), but not for devices. This may be due to the rarely serious nature of the problem. However, due to the high prevalence and moderately rated distress by users, this is important to systematically address.
- Further studies of device users should include knowledge assessment to determine how they prevent and treat skin reactions. Targeted education on prophylaxis, placement, adhesives, and device removal may reduce incidence or severity of skin reactions (9). Clinicians must be informed of options to present to patients, and research is needed to determine best practices for skin management.

\section{Insulin pump breakdown and infusion set failure in Italian children with type 1 diabetes: 1-year prospective observational study with suggestions to minimize clinical impact}

Rabbone $I^{1}$, Minuto $N^{2}$, Toni $S^{3}$, Lombardo $F^{4}$, Iafusco $D^{5}$, Marigliano $M^{6}$, Schiaffini $R^{7}$, Maltoni $G^{8}$, Frongia $A P^{9}$, Scardapane $M^{10}$, Nicolucci $A^{10}$, Cherubini $V^{11}$, Bonfanti $R^{12}$, Scaramuzza $A E^{13}$; for the Diabetes Study Group of the Italian Society of Pediatric Endocrinology and Diabetology

${ }^{1}$ Department of Paediatrics, University of Turin, Turin, Italy; ${ }^{2}$ Department of Paediatrics, IRCCS Istituto Giannina Gaslini, Genoa, Italy; ${ }^{3} J u v e n i l e$ Diabetes Center, Anna Meyer Children's Hospital, Florence, Italy; ${ }^{4}$ Department of Paediatric Sciences, University of Messina, Messina, Italy; ${ }^{5}$ Regional Center for Paediatric Diabetes "'G. Stoppoloni,", Department of Paediatrics, University of Campania "Luigi Vanvitelli," Naples, Italy; ${ }^{6}$ Regional Center for Paediatric Diabetes, Paediatric Diabetes and Metabolic Disorders Unit, University of Verona, Verona, Italy; ${ }^{7}$ Diabetes Unit, University Department of Paediatric Medicine, Bambino Gesù Children's Hospital, Rome, Italy; ${ }^{8}$ Department of Paediatrics, S.Orsola-Malpighi Hospital, Bologna, Italy; ${ }^{9}$ Unit of Paediatric Diabetes, Brotzu Hospital, Cagliari, Italy; ${ }^{10}$ CORESEARCH, Center for Outcomes Research and Clinical Epidemiology, Pescara, Italy; ${ }^{11}$ Division of Pediatric Diabetology, Department of Woman's and Children's Health, AOU G. Salesi Hospital, Ancona, Italy;

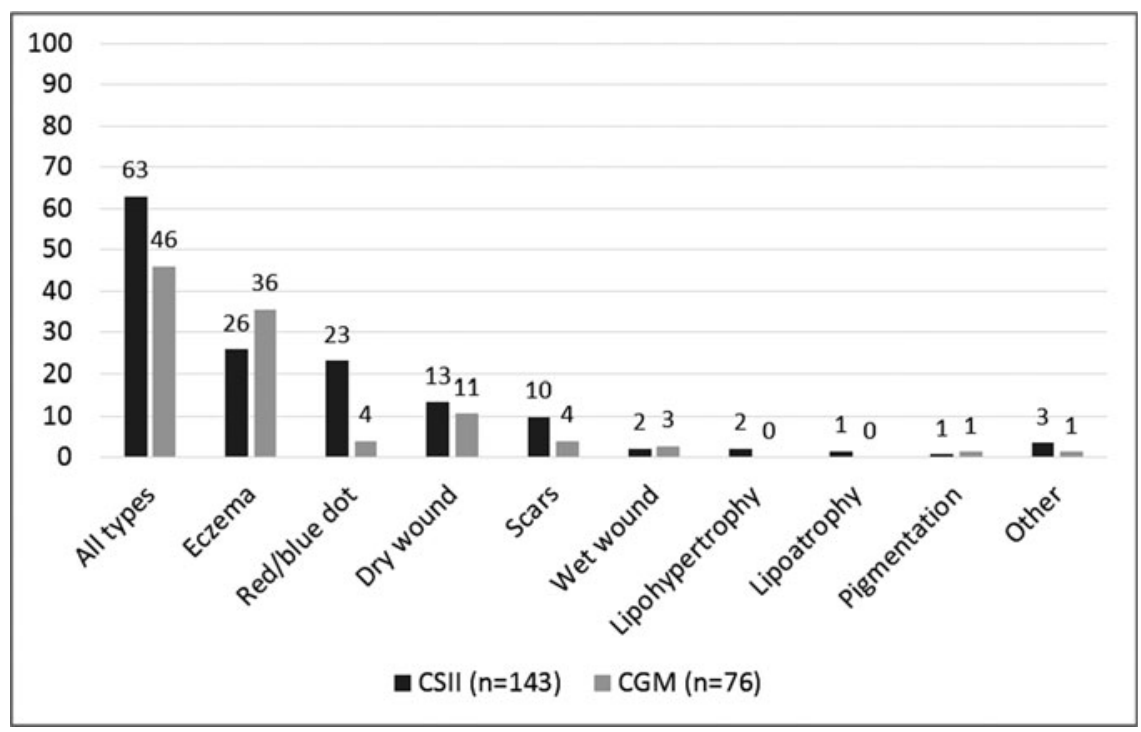

FIG. 3. Percent of participants experiencing current dermatological complications from continuous subcutaneous insulin infusion and CGM use. 
${ }^{12}$ Pediatric Diabetes and Diabetes Research Institute, San Raffaele Hospital, Milan, Italy; ${ }^{13}$ Pediatric Diabetes and Endocrinology \& Nutrition ASST Cremona, Cremona, Italy

Diabetes Obes Metab 2018; 20: 2551-2556

\section{Background}

Insulin pump set failures can result in severe hyperglycemia or diabetic ketoacidosis if undetected or mitigated. There are no prospective long-term studies characterizing the problem in individuals with diabetes.

\section{Methods}

Twenty-five pediatric diabetes centers in Italy prospectively documented insulin pump malfunctions and infusion set breakdowns for 1046 children and adolescents with T1D (50\% male, mean age $12.2 \pm 4.1$ years) using insulin pumps for mean $3.3 \pm 2.2$ years. Individuals were followed for three quarterly visits and reported number of infusion set problems and types (kinking, bubbles in tubing, set dislodgement, bleeding, occlusion, etc.) and clinical adverse events (hyperglycemia, diabetic ketoacidosis, etc.). Poisson regression was used to determine factors related to infusion set failures.

\section{Results}

The highest incidence of infusion set problems (events per person-year) was for unexplained hyperglycemia that resolved with infusion set change (8.4 events/person-year), followed by 7 for bubbles, 2.8 for cannula kinking, 2.4 for bleeding, 2 for set dislodgement, 2 for occlusion, 1.9 for tunneling (leak back), 1.8 for lipohypertrophy, and 0.3 for infection. The average incident rate ratio for individual types of infusion set problems was 4.5 failures/person-year. In multivariate analysis, risk of unexplained hyperglycemia was lower for aspart vs lispro, use of angled cannula, age $>11$ years, and catheter changes at 2 days or more. Preschoolers ages 0-6 years showed higher incidence of bubbles, unexplained hyperglycemia, and lipohypertrophy, while adolescents ages 1117 had more occlusions and tunneling. Aspart was associated with less unexplained hyperglycemia and bubbles, whereas glulisine was associated with higher risk of occlusions and lipohypertrophy. Angled cannulas were associated with dramatic decreases in nearly all of the infusion set problems studied compared with 90-degree cannulas, however, they presented a nearly 3-fold increase in infection risk. Lipohypertrophy was significantly associated with higher HbAlc.

\section{Conclusion}

In a prospective long-term study in children and adolescents using insulin pump, there is a relatively low average rate of infusion set problems; however, it varies widely by type of problem. Lipohypertrophy was the only issue associated with higher $\mathrm{HbA} 1 \mathrm{c}$.

\section{Comments}

Previous studies have demonstrated infusion set failures to be a persistent problem in both children and adults with metabolic and nonmetabolic consequences (10-
12). In 2018, despite the multitude of advancements in other technological fields, infusion sets remain unchanged, causing the same issues for patients that have been present for 30 years: nonspecific set failure (hyperglycemia), kinking, bubbles, bleeding, site dislodgement, occlusion, leak-back, lipohypertrophy, and infection. This Italian study in children and adolescents cements the persistence of these issues and provides a longer duration (1 year) of data than any previous study. It is important to consider these points:

- The average incidence rate for infusion set problems was calculated by the authors to be 4.5 failures/ person-year. More important than this, however, is the cumulative incidence rate of all the problems. Simply adding the reported overall incidence rates of the nine main problems listed leads to a cumulative incidence of over two infusion set issues each month. This is clinically significant and distressing for patients and should prompt discussion among clinicians and patients to identify solutions.

- Lipohypertrophy was the only issue that led to HbA1c changes in multivariate analysis (mean change 0.2 $\mathrm{mmol} / \mathrm{mol}, P=0.003$ ). This confirms previous findings of glycemic change with lipohypertrophy (13).

- Angled cannulas appeared to substantially reduce incidence of all infusion set problems, with the exception of infection. How often are these used in clinical practice, and why are they not used more? It is unknown how pump trainers or clinicians decide on infusion set type for patients, and this should be studied. Although children may find angled cannulas more objectionable due to size and perceived pain, perhaps education regarding benefits could increase use in this population.

- Similar studies should be conducted using steel cannulas as well, as they are not included in this analysis.

\section{Impact of targeted education on managing warning and error signals by children and adolescents with type 1 diabetes using the Accu-Chek Combo Insulin Pump System}

\author{
Deeb A, Qahtani NA, Ali AA, Akle M
}

Department of Paediatric Endocrinology, Mafraq Hospital, AbuDhabi, United Arab Emirates

Ann Pediatr Endocrinol Metab 2017; 22: 170-175

\section{Background}

Insulin pumps are equipped with warning, safety, and error alerts that require troubleshooting or user involvement. Pump manuals are often difficult to navigate due to length and complexity, making it difficult to quickly locate relevant information. This study provided targeted education to insulin pump users to understand and attend to pump alarms.

\section{Methods}

Pediatric patients using the Accu-Chek insulin pump for $>6$ months were recruited for this study, which consisted of 
two visits to the diabetes clinic. In the first visit, warning and error alerts were downloaded from the pump, assessed for frequency and type, and discussed with participants. An education/study team consisting of a diabetes educator, psychologist, and endocrinologist reviewed the types of alerts and troubleshooting steps with the individuals, providing verbal and written instructions. A second visit occurred 3 months later when interim alerts and alarms were tabulated and categorized. At both visits, participants filled out questionnaires about their understanding of pump alerts.

\section{Results}

A total of 21 participants (14 female) with T1D were enrolled with median age of 13 (range 7-18) years. Mean pump use was 2.5 years. Across all subjects, the most commonly reported pump warnings were "low cartridge" (40\%), "bolus cancelled" (31\%), and "battery low" (29\%). Common error alerts included "occlusion (46\%), "power interrupted" (18\%), and "battery empty" (13\%). Between visit 1 and visit 2 , pump warnings significantly reduced $42 \%$ from 213 alerts to 122 alerts $(P<0.001)$ and error alerts decreased $61 \%$ from 148 to $57(P=0.04)$. Participants indicated increased knowledge on understanding warning and error alerts after the education session, with questionnaire scores rising significantly $(P=0.02)$.

\section{Conclusion}

Targeted education on insulin pump alerts decreased the number of system alerts and increased subjective understanding of the pump alerts by the user.

\section{Comment}

The literature is profoundly lacking in educational interventions for diabetes technology. This is a straightforward study from the United Arab Emirates that examines the impact of continuous subcutaneous insulin infusion (CSII) alarm/alert education. By tailoring education on alerts to patients based on their alarm history, the study team significantly reduced alerts and alarms by half. Although a small study $(n=21)$, the clinical implications are clear:

- We can impact our patients' device experience by taking the time to assess problems (alert history, for example). Discussing the meaning of alerts and how to avoid/troubleshoot them in plain language can be tremendously helpful. The authors rightly suggest that user manuals are cumbersome and difficult to understand. Clinician-patient conversation may dramatically clarify and distill the most critical information for the patient.

- Education interventions like this should be studied with CGM and automated insulin technologies. More sophisticated and combination devices will likely increase alert frequency and risk of alarm fatigue. Targeted education provides a simple intervention for a growing concern.

\section{ACCESS AND ECONOMICS}

\section{Insulin regimens for newly diagnosed children with type 1 diabetes mellitus in Australia and New Zealand: a survey of current practice}

Selvakumar $D^{1}$, Al-Sallami $H^{1}$, de Bock $M^{2}$, Ambler $G R^{3}$, Benitez-Aguirre $P^{3}$, Wiltshire $E^{4}$, Tham $E^{5}$, Simm $P^{6}$, Conwell $L S^{7,8}$, Carter $P J^{9}$, Albert BB ${ }^{9}$, Willis $J^{10}$, Wheeler $\mathrm{BJ}^{11}$; on behalf of the Paediatric Society of New Zealand Diabetes Clinical Network

${ }^{1}$ School of Pharmacy, University of Otago, Dunedin, New Zealand; ${ }^{2}$ Department of Paediatric and Child Health, Princess Margaret Hospital, Perth, Western Australia, Australia; ${ }^{3}$ Institute of Endocrinology and Diabetes, Children's Hospital at Westmead and University of Sydney, Sydney, New South Wales, Australia; ${ }^{4}$ Department of Paediatrics and Child Health, University of Otago, Wellington, New Zealand; ${ }^{5}$ Department of Endocrinology and Diabetes, Women's and Children's Hospital, Adelaide, South Australia, Australia; ${ }^{6}$ Department of Endocrinology and Diabetes, Royal Children's Hospital Melbourne, Melbourne, Victoria, Australia; ${ }^{7}$ School of Medicine, University of Queensland, Brisbane, Queensland, Australia; ${ }^{8}$ Department of Endocrinology and Diabetes, Lady Cilento Children's Hospital, Brisbane, Queensland, Australia; ${ }^{9}$ Starship Paediatric Diabetes and Endocrinology, Starship Children's Health, Auckland, New Zealand; ${ }^{10}$ Don Beaven Medical Research Centre, Christchurch, New Zealand; ${ }^{11}$ Department of Women's and Children's Health, University of Otago, Dunedin, New Zealand

\section{J Paediatr Child Health 2017; 53: 1208-1214}

\section{Background}

There is no expert consensus on ideal insulin regimen for new onset T1D, as there are many factors to consider, including age, access to supplies, and comfort of provider and family with insulin pumps vs injections. The current study explores trends in insulin pump initiation vs insulin injection in new onset T1D diagnosis in Australia and New Zealand.

\section{Methods}

This is a cross-sectional survey of diabetes clinicians in Australia and New Zealand identified through pediatric diabetes professional organizations. Providers were split into five geographical regions. Participants were queried on professional practices with new onset T1D including insulin regimen and other management choices.

\section{Results}

A total of 100 clinicians were surveyed in this study: $43 \%$ from New Zealand and $57 \%$ from Australia. The majority $(71 \%)$ were endocrinologists, and $29 \%$ were general pediatricians. In Australia, the largest proportion of new onset T1D was treated with multiple daily injections (MDI) (47\% of toddlers $<2$ years old, $64 \%$ of children $2-10$ years, and $93 \%$ of adolescents $>10$ years old). CSII was considered for $38 \%$ of toddlers ( $<2$ years old), but only considered in $9 \%$ of older children and $7 \%$ of adolescents. In New Zealand, conventional twice-daily insulin dosing was preferred for toddlers 
(71\%) and children ages $2-10$ years $(69 \%)$, and MDI was preferred for adolescents ages $>10$ years $(75 \%)$. Clinicians in both countries (64\% of Australian and $90 \%$ of New Zealand clinicians) identified access as a primary obstacle in insulin pumps at new onset. The $36 \%$ in Australia who indicated some accessibility identified private health insurance (79\%), donated insulin pumps (14\%), and out-of-pocket payment $(7 \%)$ as potential options. In New Zealand, outof-pocket payment was the only identified option for new onset T1D.

\section{Conclusion}

Access to insulin pumps at new onset T1D is a challenge for clinicians in Australia and New Zealand and limits options for insulin modalities at diabetes onset. Insulin regimens vary greatly between clinicians, region, and country. Consensus guidelines may help standardize new onset insulin practices in the future.

\section{Comments}

Access and economic research have broad applicability in addition to country-specific implications. The story of prescribing practices for insulin pumps/MDI in new onset T1D in Australia and New Zealand is one of access but also of evidence. Although this article was initially selected for its applicability to insulin pumps in new onset T1D, the broader point to note is that intensive insulin therapy is not something to be taken for granted. The following points were remarkable:

- The authors describe the surprising lack of consensus in pediatric diabetes organizations on intensive therapy vs conventional twice daily insulin for T1D in the pediatric population. This is the more urgent implication of this article than CSII vs MDI preference for new onset T1D.

- Insulin pump prescription was vastly different between New Zealand and Australia, with New Zealand physicians reporting nearly no insulin pumps for T1D onset, and Australian physicians prescribing insulin pump for $38 \%$ of toddlers, $9 \%$ of older children, and $7 \%$ of adolescents. In addition to clinical evidence concerns, this was largely attributed to financial access.

- Prescribing practices for diabetes technology (and any variation of insulin regimens) is a fine balance between clinical recommendations, funding and availability, and access to resources to support the therapy decisions. It is a constellation of factors that drive clinical practice, and increasing technology uptake will need to involve systematic drivers, clinician education, increased reimbursement, and an exploration of clinical support to enable technology integration.

\section{Cost-effectiveness of continuous glucose monitoring for adults with type 1 diabetes compared with self-monitoring of blood glucose: the DIAMOND randomized trial}

Wan $W^{1}$, Skandari $M R^{1}$, Minc $A^{1}$, Nathan $A G^{1}$, Winn $A^{2}$, Zarei $P^{l}$, O'Grady $M^{3}$, Huang $E S^{l}$
${ }^{1}$ Section of General Internal Medicine, University of Chicago, Chicago, IL; ${ }^{2}$ School of Pharmacy, Medical College of Wisconsin, Milwaukee, WI; ${ }^{3}$ National Opinion Research Center, University of Chicago, Chicago, IL

Diabetes Care 2018; 41: 1227-1234

\section{Background}

In addition to the clinical benefits of CGM, costeffectiveness is an important factor in access and utilization of the technology. This study evaluated the societal costeffectiveness of CGM in individuals with T1D using multiple daily injections.

\section{Methods}

This is a cost-effectiveness analysis of the 158 trial participants in the Multiple Daily Injection and Continuous Glucose Monitoring in Diabetes (DIAMOND) trial. The DIAMOND trial included 2:1 randomization to CGM or SMBG for adult participants with $\mathrm{HbA} 1 \mathrm{c} \geq 7.5 \%$ using MDI, and trial duration was 6 months. Information on health-related QOL, health-care service utilization, medications and supplies, work productivity, and self-management time were collected at baseline and 6 months. Within-trial and lifetime cost-effectiveness analyses were performed using a Sheffield T1D policy model (modified to include non-severe hypoglycemic events, QOL, and cost effects) to estimate complications related to diabetes. The analysis used the societal perspective and discounted costs and quality-adjusted life years (QALYs) at 3\% per year. The primary outcome was cost per QALY.

\section{Results}

During the 6-month trial, QALYs were not significantly different between the CGM group and SMBG group $(P=0.61)$. Cost for the CGM group was $\$ 11,032$ (USD) and $\$ 7,236$ for the SMBG group for 6 months. The cost difference was only in the device cost and not in diabetes care, medical visits, or work productivity. In the 6-month trial, the CGM group had a higher reduction in HbA1c $(P>0.01)$, lower daily test strip use $(P=0.04)$, and less incidence of nonsevere hypoglycemia. In the lifetime cost-analysis, CGM was predicted to increase QALYs by 0.54 over SMBG with the incremental cost-effectiveness ratio of $\$ 98,108$ per QALY. Sensitivity analysis showed that if real-world CGM use extended sensor life from 7 to 10 days, the cost-effectiveness ratio could decrease as low as \$33,459/QALY for Dexcom G4 and \$41,464 per QALY for Dexcom G5. The analysis was also highly sensitive to expected $\mathrm{HbA1c}$ benefit.

\section{Conclusion}

The lifetime cost-effectiveness analysis of CGM in adults with T1D using MDI showed that CGM is cost-effective at a willingness-to pay threshold of $\$ 100,000$ per QALY.

\section{Comments}

Cost-effectiveness analyses are important for the expansion of payment for diabetes technologies. Various governmental and private payment systems use QALYs 
as a metric to determine whether a therapy is "worth it" to their reimbursement system. Large-scale randomized trials like the DIAMOND study have provided robust data for cost-effectiveness analysis of CGM in various populations, in this case individuals with T1D on MDI without an immediate clinical indication for CGM. To note:

- This is the first major trial of CGM representing MDI patients with newer generation devices. This is an important population for analysis considering the majority of individuals with T1D worldwide are on injections and not on insulin pumps

- The primary costs in this analysis were the CGM components themselves. The sensitivity analysis illustrates that optimizing CGM wear from 7 to 10 days would more than halve the cost per QALY, potentially opening up reimbursement pathways that were previously denied. In addition to the QOL and user burden perspective, there is an economic imperative to make CGM components last longer, reducing cost and increasing access.

\section{Cost-effectiveness of insulin pumps compared with multiple daily injections both provided with structured education for adults with type 1 diabetes: a health economic analysis of the Relative Effectiveness of Pumps over Structured Education (REPOSE) randomised controlled trial}

Pollard DJ $J^{1}$, Brennan $A^{1}$, Dixon $S^{l}$, Waugh $N^{2}$, Elliott $J^{3}$, Heller $S^{3}$, Lee $E^{4}$, Campbell $M^{1}$, Basarir $H^{5}$ White $D^{4}$; on behalf of the REPOSE group

${ }^{1}$ School of Health and Related Research (ScHARR), University of Sheffield, Sheffield, UK; ${ }^{2}$ Population Evidence and Technologies, Warwick Medical School, University of Warwick, Coventry, UK; ${ }^{3}$ Academic Unit of Diabetes, Endocrinology and Metabolism, Department of Oncology and Metabolism, School of Medicine and Biomedical Sciences, University of Sheffield, Sheffield, UK; ${ }^{4}$ Clinical Trials Research Unit, School of Health and Related Research (ScHARR), University of Sheffield, Sheffield, UK; ${ }^{5}$ RTI Health Solutions, Manchester, UK

BMJ Open 2018; 8: e016766

\section{Background}

In England, $11.7 \%$ of individuals with T1D are treated with insulin pump. The Relative Effectiveness of Pumps over Structured Education (REPOSE) study was conducted to assess the benefit of insulin pumps compared with MDI in adults with T1D, both arms of which additionally received structured education. This manuscript presents a costeffectiveness analysis of pumps vs MDI.

\section{Methods}

The REPOSE study was conducted at eight centers in England and Scotland in 267 individuals with T1D who did not have an immediate clinical need for an insulin pump. Cost-effectives analysis was performed on the bootstrapped data from 260 REPOSE participants that had HbA1c data. The Sheffield Type 1 Diabetes Policy Model was used to estimate lifetime incidence of complications related to diabetes, resources used, and effects on QALYs. The analyses used a National Health Service and societal perspective and a discount rate of $3.5 \%$ per year for costs and QALYs. Primary outcomes were incremental costs, QALYs gained, and incremental cost-effectiveness ratios.

\section{Results}

In the lifetime cost analysis, insulin pumps + education were associated with $£ 18,852$ (95\% CI $£ 6175-£ 31645$ ) increase in cost, and a 0.13 increase in QALYs $(95 \%$ CI0.70.96). Insulin pumps + structured education was estimated to contribute to $\mathrm{HbAlc}$ trends increasing $0.052 \%$ per year compared with MDI + structured education increasing $0.054 \%$ per year. The base care incremental cost-effectiveness ratio was $£ 142,195$ per QALY gained over MDI + education. The ratio was sensitive to cost of insulin pumps and consumables and expected $\mathrm{HbAlc}$ change. The lowest incremental costeffectiveness ratio found in sensitivity analysis was with pump supply costs being halved ( $\$ 31,747$ per QALY).

\section{Conclusion}

Since the National Institute for Health and Care Excellence threshold for cost-effectiveness in the United Kingdom is typically below $£ 30,000$ per QALY, there is no likely scenario in this analysis that would suggest insulin pump therapy being cost-effective for adults with T1D who are not otherwise indicated for insulin pump.

\section{Comments}

The REPOSE study is interesting because it examines the relative impact of insulin pumps over MDI in adults with T1D who "did not have a clinical indication to receive a pump immediately, as determined by the investigator, or did not have a strong preference to receive a pump." In this particular population, within the United Kingdom societal perspective, insulin pumps were not cost-effective. To consider:

- For simplicity and cost sake, patients who do not have a preference for MDI vs insulin pump (nor a clinical need) should not be pushed to start insulin pump therapy. For this adult population without a clinical indication for insulin pump, health benefits were incremental and lifetime costs high.

- As diabetes technology increases in usefulness (automated insulin, predictive low-glucose suspend, etc.), new evaluations of cost-effectiveness will need to be done, and new populations will need to be considered for diabetes technology benefit. It is difficult to extrapolate results from one device to another. Diabetes devices have a wide variety of uses, and device specifics (usability, utility, human factors) will inform appropriate characteristics for candidacy.

- The population in this study was chosen by the investigators, which parallels the provider role in clinical practice. Research needs to continue to inform 
who is "clinically indicated" for diabetes technology and who is not. Although diabetes technology should be open for all, it is most effectively used by those most likely to benefit. This will likely be different by device class (pump vs CGM vs automated insulin, etc.).

- A hidden brilliance in this study is the use of educational intervention in both the control and insulin pump arm. Although the study was not measuring the impact of the educational intervention, the authors rightly point out that the clinical initiation of insulin pump therapy usually comes with additional diabetes education that patients may not otherwise experience. By providing both control and pump patients with similar levels of additional structured education, the intervention of insulin pump is better measured and outcomes attributable to insulin pump only.

\section{Author Disclosure Statement}

L.M.'s institution has received funding from Medtronic, Dexcom, Insulet, Roche, and Bigfoot Biomedical. She has received consulting fees from Tandem Diabetes, Capillary Biomedical, and Clinical Sensors.

\section{References}

1. Kowalski AJ. Taking do-it-yourself artificial pancreas systems mainstream. JDRF October 18, 2017. www.jdrf.org/ blog/2017/10/18/taking-artificial-pancreas-systems-mainstream (Accessed July 11, 2018).

2. Wong JC, Neinstein AB, Spindler M, Adi S. A minority of patients with type 1 diabetes routinely downloads and retrospectively reviews device data. Diabetes Technol Ther 2015; 17: 555-562.

3. Miller KM, Foster NC, Beck RW, Bergenstal RM, DuBose SN, DiMeglio LA, etc.; T1D Exchange Clinic Network.
Current state of type 1 diabetes treatment in the U.S.: updated data from the T1D Exchange clinic registry. Diabetes Care 2015; 38: 971-978.

4. Berg AK, Nørgaard K, Thyssen JP, Zachariae C, Hommel E, Rytter K, Svensson J. Skin problems associated with insulin pumps and sensors in adults with type 1 diabetes: a cross-sectional study. Diabetes Technol Ther 2018;20:475482.

5. Schober E, Rami B. Dermatological side effects and complications of continuous subcutaneous insulin infusion in preschool-age and school-age children. Pediatr Diabetes 2009; 10: 198-201.

6. American Diabetes Association [ADA]. 9. Cardiovascular disease and risk management. Diabetes Care 2018; 41: S86-S104.

7. ADA. 10. Microvascular complications and foot care. Diabetes Care 2018; 41: S105-S118.

8. ADA. 4. Lifestyle management. Diabetes Care 2018; 41: S38-S50

9. Messer LH, Berget C, Beatson C, Polsky S, Forlenza GP. Preserving skin integrity with chronic device use in diabetes. Diabetes Technol Ther 2018; 20: S254-S264.

10. Wheeler BJ, Heels K, Donaghue KC, Reith DM, Ambler GR. Insulin pump-associated adverse events in children and adolescents-a prospective study. Diabetes Technol Ther 2014; 16: 558-562.

11. Ross PL, Milburn J, Reith DM, Wiltshire E, Wheeler BJ. Clinical review: insulin pump-associated adverse events in adults and children. Acta Diabetol 2015; 52: 1017-1024.

12. Pickup JC, Yemane N, Brackenridge A, Pender S. Nonmetabolic complications of continuous subcutaneous insulin infusion: a patient survey. Diabetes Technol Ther 2014; 16: 145-149.

13. Famulla S, Hövelmann U, Fischer A, Coester HV, Hermanski L, Kaltheuner M, et al. Insulin injection into lipohypertrophic tissue: blunted and more variable insulin absorption and action and impaired postprandial glucose control. Diabetes Care 2016; 39: 1486-1492. 